\title{
MVPA Analysis of Intertrial Phase Coherence of Neuromagnetic Responses to Words Reliably Classifies Multiple Levels of Language Processing in the Brain
}

\author{
DiD Mads Jensen, ${ }^{1}$ Rasha Hyder, ${ }^{1}$ and Yury Shtyrov ${ }^{1,2}$
}

https://doi.org/10.1523/ENEURO.0444-18.2019

${ }^{1}$ Center of Functionally Integrative Neuroscience (CFIN), Department of Clinical Medicine, Aarhus University, 8000 Aarhus, Denmark and ${ }^{2}$ Laboratory of Behavioural Neurodynamics, St. Petersburg State University, St. Petersburg, 199034, Russia

\begin{abstract}
Neural processing of language is still among the most poorly understood functions of the human brain, whereas a need to objectively assess the neurocognitive status of the language function in a participant-friendly and noninvasive fashion arises in various situations. Here, we propose a solution for this based on a short task-free recording of MEG responses to a set of spoken linguistic contrasts. We used spoken stimuli that diverged lexically (words/pseudowords), semantically (action-related/abstract), or morphosyntactically (grammatically correct/ungrammatical). Based on beamformer source reconstruction we investigated intertrial phase coherence (ITPC) in five canonical bands ( $\alpha, \beta$, and low, medium, and high $\gamma$ ) using multivariate pattern analysis (MVPA). Using this approach, we could successfully classify brain responses to meaningful words from meaningless pseudowords, correct from incorrect syntax, as well as semantic differences. The best classification results indicated distributed patterns of activity dominated by core temporofrontal language circuits and complemented by other areas. They varied between the different neurolinguistic properties across frequency bands, with lexical processes classified predominantly by broad $\gamma$, semantic distinctions by $\alpha$ and $\beta$, and syntax by low $\gamma$ feature patterns. Crucially, all types of processing commenced in a near-parallel fashion from $\sim 100 \mathrm{~ms}$ after the auditory information allowed for disambiguating the spoken input. This shows that individual neurolinguistic processes take place simultaneously and involve overlapping yet distinct neuronal networks that operate at different frequency bands. This brings further hope that brain imaging can be used to assess neurolinguistic processes objectively and noninvasively in a range of populations.
\end{abstract}

Key words: language; magnetoencephalography (MEG); multivariate pattern analysis (MVPA); oscillations; lexical access; semantics; morphosyntax

\section{Significance Statement}

In an MEG study that was optimally designed to test several language features in a non-attend auditory paradigm, we found that, by analyzing cortical source-level intertrial phase coherence (ITPC) in five canonical bands $(\alpha, \beta$, and low, medium, and high $\gamma$ ) with machine-learning classification tools [multivariate pattern analysis (MVPA)], we could successfully classify meaningful words from meaningless pseudowords, correct from incorrect syntax, and semantic differences between words, based on passive brain responses recorded in a task-free fashion. The results show different time courses for the different processes that involve different frequency bands. It is to our knowledge the first study to simultaneously map and objectively classify multiple neurolinguistics processes in a comparable manner across language features and frequency bands. 


\section{Introduction}

A neuropsychological assessment of the neurologic and/or cognitive status of a subject or patient is a routine required in a variety of situations. This typically involves elaborate behavioral tests aimed, for instance, at evaluating the extent of developmental disorders, assessing the level of neurodegenerative impairment, neurologic damage after a head injury, screening for hearing loss, etc. To perform such tests successfully, the subject, on the one hand, must typically have a reasonably clear understanding of what they have to do in a particular procedure; on the other hand, they must also be able to communicate their responses to a given task, by giving, e.g., a manual, facial or oral response. This raises the problem of uncooperative subjects, such as those suffering from neurologic/organic brain disorders or mental illnesses that cause patients to become unresponsive. A brain-damaged person may be unable to respond verbally because of a collateral lesion-related injury (such as akinetic mutism, paralysis, aphasia). A young child with a developmental disorder may be unable or unwilling to communicate their reaction overtly. A locked-in patient, although not necessarily unconscious, is unable to respond due to the total loss of their motor control function (Ragazzoni et al., 2000; Laureys et al., 2004). On the other hand, an undetected language impairment could have drastic consequences for (mis)diagnosis of language-unrelated disturbances (Majerus et al., 2009). A range of such situations is wide and they create a substantial challenge for diagnosis and assessment of performance, development or recovery in various groups. Clearly, techniques that could reveal the brain processing of language without relying on the individual's overt behavior would be helpful in such cases.

Fast-paced development of non-invasive neuroimaging techniques in recent years has given hopes of assessing the brain status of various cognitive functions objectively, even when overt response may not be possible. Language is a complex phenomenon that, to make a coherent whole, requires multiple information types involving different specific features and properties that rapidly unfold in time with a fine temporal structure and remarkable speed

Received November 7, 2018; accepted June 28, 2019; First published August 5, 2019.

The authors declare no competing financial interests.

Author contributions: M.J. and Y.S. contributed unpublished reagents/anaIytic tools; M.J. analyzed data; M.J. and Y.S. wrote the paper; R.H. and Y.S. designed research; R.H. performed research.

This work was supported by the Danish Council for Independent Research (DFF, grant 6110-00486), Lundbeck Foundation (grants R164-2013-15801, R140-2013-12951), Central Jutland Regional Government (MINDLab infrastructure grant), RF Government (grant contract No 14.W03.31.0010), and Aarhus University.

Acknowledgment: We thank Andreas Højlund, Karen Østergaard, Christelle Gansonre, Alina Leminen, Eino Partanen, Nikola Vukovic, Lilli Kimppa, Christopher Bailey, and Britta Westner for their help at different stages of this work.

Correspondence should be addressed to Mads Jensen at mads@cfin.au.dk. https://doi.org/10.1523/ENEURO.0444-18.2019

Copyright (C) 2019 Jensen et al.

This is an open-access article distributed under the terms of the Creative Commons Attribution 4.0 International license, which permits unrestricted use, distribution and reproduction in any medium provided that the original work is properly attributed.
(Friederici, 2002, 2011; Shtyrov, 2010). This, in turn, suggests that for the capturing of cerebral processing of linguistic information, a temporally-resolved neuroimaging method is needed that could faithfully track the dynamic neural activity during language comprehension (Hagoort, 2008). At present, two main techniques are able to provide such high temporal resolution: MEG and EEG, both capable of registering mass neural activity on a millisecond scale. Whereas the two methods are highly similar, MEG has a certain advantage in the ease of modeling underlying cortical activity, owing to unhindered spreading of magnetic fields (but not electric currents) through the head and skull tissues (Baillet et al., 2001).

A body of electrophysiological research done in EEG and MEG using various linguistic materials has provided a rich picture of linguistic processing in the brain. Perhaps the most well-known neurophysiological response to language stimuli is the so-called N400 (Kutas and Hillyard, 1980), usually seen as an index of (lexico)semantic processes and peaking at $\sim 400 \mathrm{~ms}$. Syntactic (grammatical) processing has been associated with an early ELAN (early left anterior negativity) response reflecting the stimulus' grammaticality from $\sim 100 \mathrm{~ms}$ (Neville et al., 1991; Friederici et al., 1993), as well as later frontal negativities (LAN) with longer latencies (Münte et al., 1998; Gunter et al., 2000) and the P600, a late positive shift (Osterhout et al., 1994).

Most of these responses (except, to an extent, ELAN) still require at least a degree of overt attention or even focused task performance from the subject. In terms of assessing linguistic processing in a more task-free fashion, a number of studies have attempted to use the socalled passive paradigms, in which the subjects are presented with linguistic contrasts without having to perform an overt task and are usually distracted from the auditory input by a video or another unrelated activity (Pulvermüller and Shtyrov, 2006; Näätänen et al., 2007). A series of studies using this approach established MEG/ EEG correlates of automatic linguistic access including phonological, lexical, semantic, and syntactic levels of information processing (Shtyrov, 2010). These have shown that information-specific linguistic activations can be recorded noninvasively without an explicit task or even an instruction to focus on the speech input. For instance, meaningful native words show stronger activation in the core language system than meaningless acoustically similar pseudowords, which putatively indicates activation of word-specific memory traces (Pulvermüller et al., 2001). Furthermore, these activations show both semantic specificity (e.g., by involving motor cortex activation and deactivation when presenting action-related words; Hauk et al., 2008; Moseley et al., 2013; Shtyrov et al., 2014) and sensitivity to grammatical properties (ELAN-like activity in response to syntactic violations; Hasting et al., 2007).

Such studies of neurolinguistic processing typically focus on one single process (e.g., syntax or semantics) at a time. For this approach to be more practically-oriented, it would seem essential to develop a task-free paradigm that can assess multiple types of linguistic information processing in a single short participant-friendly session. 
Furthermore, previous studies have mostly investigated neurolinguistic processes using ERP/ERFs, that is, evoked activity in a rather narrow frequency band (typically 1-30 Hz), and it would thus appear advantageous to open up the frequency spectrum to maximize possibilities for registering brain reflections of language. Oscillations in different frequency bands have been shown to reflect multiple cognitive processes and are considered to be a vehicle for neural communication (Varela et al., 2001; Fries, 2005). Different frequency bands have been ascribed different functional roles in the neocortical information processing (Sauseng and Klimesch, 2008; Cannon et al., 2014), where higher bands could be related to local computations (Buzsáki and Silva, 2012; Buzsáki and Schomburg, 2015) and lower bands to longer-range connectivity (Von Stein and Sarnthein, 2000). That different neurolinguistic processes involve different frequency bands has been shown in a range of studies. For instance, Bastiaansen and Hagoort (2006) argued that retrieval of lexical information and unification of semantic and syntactic information are underpinned by different oscillatory networks, while Kösem and van Wassenhove (2017) suggested that $\theta$ oscillations $(3-8 \mathrm{~Hz}$ ) are related to acoustic properties. Teng et al. (2017) shows that humans can track spoken language dynamics at both $\theta$ and $\gamma$ oscillations. Using electrocorticography (ECoG), Towle et al. (2008) have shown an increase in high $\gamma(70-100 \mathrm{~Hz})$ in relation to hearing a word compared to a tone. Luo and Poeppel (2007) showed that phase information in the oscillatory dynamics can track and discriminate spoken sentences. However, although the interest in timefrequency analysis of electrophysiological data has been steadily rising in recent years, language-related oscillatory dynamics still remains relatively unexplored, with most studies in the field focusing on evoked potentials/fields instead.

In an attempt to close the gap between these research strands and at the same time bridge this research with applied/clinical needs, we set out to combine these different approaches and designed a simple short paradigm that simultaneously includes lexical, semantic, and syntactic contrasts, to assess different levels of linguistic processing in a single session. This paradigm is tested here by recording MEG responses in a sample of healthy adult participants as a first step to establishing its applicability. The participants were presented with spoken stimuli, which were either meaningless pseudowords or meaningful words with different semantics (action-related verb vs concrete visual noun) and that either could be syntactically correct or included a morphosyntactic stem-affix violation. These were presented without any stimulus-related task, while the subjects' attention was diverted away from the auditory stimulus stream.

Furthermore, in order not to restrict our results to narrow-band evoked activity, we analyzed activation in a wide range of frequency bands, from $\alpha$ to high $\gamma$. We focused on the phase part of the oscillatory activity, as phase synchrony has been theorized to be directly related to neural computations (Fries, 2005; Palva et al., 2005; Lopes da Silva, 2006; Varela et al., 2001). To quantify coordinated neural phase activity related to neurolinguistic processing, we determined phase synchrony by analyzing intertrial phase coherence (ITPC) of the MEG responses. ITPC is a measure of how aligned the phase angles over individual trials are. It therefore provides information about mass-synchronized neural activation (and in this sense is to a degree similar to ERP/ERF) without restricting it to a specific power peak or band. ITPC can inform us about the phase synchrony over trials; i.e., if ITPC is high, it means that the phases become aligned when performing a particular perceptual or cognitive computation. We investigated the ITPC in different frequency bands allowing for a comprehensive assessment of the neurolinguistic dynamics. To gain anatomic specificity, single-subject MRls were used to model cortical source activity, and ITPC values were calculated in individual source space.

Given the vast amount of information about neural activity created by analyzing the ITPC across both time and frequency bands, we used multivariate pattern analysis (MVPA) for an unbiased statistical assessment of the data (Bzdok et al., 2018), where by "unbiased" we mean that that the researcher does not have to choose when and where to test for differences. MVPA (for more details, see Materials and Methods) is a machine learning technique based on predicting data rather than on parameter estimation (as used in traditional factorial analyses, e.g., ANOVA or $t$ tests). The MVPA algorithm will first try to extract a pattern from a subset of the data, which can then be used to predict new, previously unseen data. This allows for a data-driven analysis without a priori hypotheses about spatial or temporal location of the signal of interest. For instance, we might allow the decoding algorithm to train itself on MEG recording trials of meaningful versus meaningless word form stimuli (i.e., find specific patterns of features in the data most valuable for detecting word-pseudoword differences) and then ask whether it can correctly predict the same distinction in a different data subset; this can be done both within and across subjects. Crucially, such an algorithm, if successful, may in principle be used to estimate data predictability in one subject (e.g., patient) from another one (e.g., healthy norm) and thus determine both normal functionality and functional abnormalities, or assess the same or different states of a particular processing system in the same individual.

In sum, we present here a short ( $<30 \mathrm{~min}$ ) task-free paradigm in which auditory linguistic stimuli are presented to MEG subjects, without explicitly requiring their attention, and the resulting data are analyzed using MVPAbased machine learning algorithm to classify brain responses in different frequency bands as reflecting meaningful lexical input as well as its semantic and syntactic properties.

\section{Materials and Methods}

\section{Participants}

The experiment was conducted according to the principles of the Helsinki Declaration and was approved by Central Jutland Region Committee on Health Research 


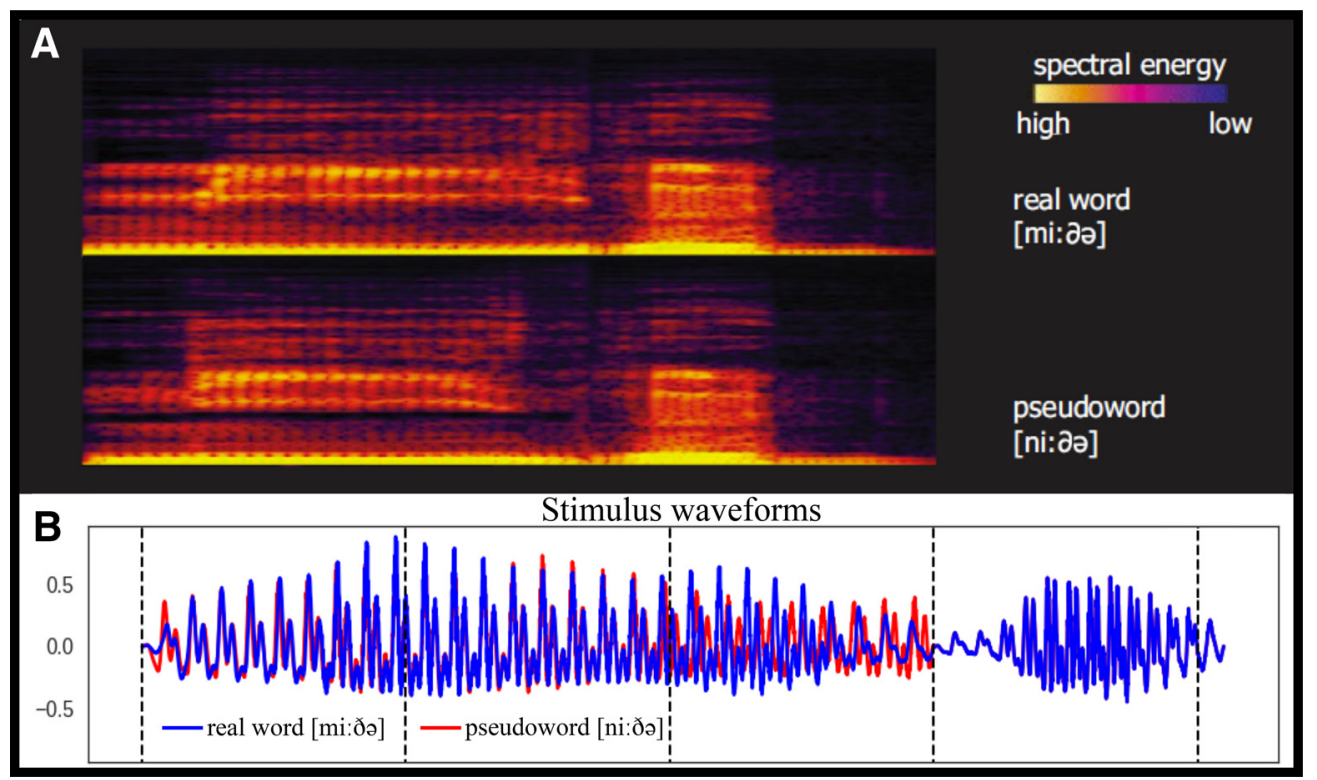

Figure 1. A, Examples of spectrograms of spoken stimuli used in the experiment (adapted from Gansonre et al., (2018)). B, Examples of waveforms plotted on top of each other.

Ethics. MEG data were acquired in seventeen healthy right-handed (handedness assessed using Oldfield, 1971) native speakers of Danish (age range 18-27 years, 12 females) with normal hearing and no record of neurologic impairments. All participants gave written consent before the start of the experiment and received payment for their participation.

\section{Stimuli}

Since we wished to address a range of different neurolinguistic processes, those at lexical, semantic, and syntactic levels, we chose stimulus items which could enable us to contrast a combination of different linguistic phenomena while controlling for acoustic features (for examples of the stimuli used, see Fig. 1). To this end, we followed a previously suggested strategy (Gansonre et al., 2018) and selected a set of spoken Danish-language stimuli which (1) belonged to different lexical and semantic categories (action-related verb, abstract verb, objectrelated noun and meaningless pseudoword), (2) were close in terms of phonology so we could compare them directly with minimal acoustic/phonetic confounds, and which (3) could be modified morpho-syntactically in the exact same way and nonetheless exhibit different linguistic properties (i.e., grammatically correct vs incorrect) such that we could test the very same contrasts in different linguistic contexts.

These requirements led to the choice of four main base stimuli: bide ([biðə], to bite), gide ([giðə], to bother), mide ([miðə], a mite), *nide ([niðə], *pseudoword). These were presented as such. Note that they have identical CVCV phonological structure and only differ in the first consonant. The second syllable [ðə], which allows recognition of the lexical items, is the same across all items. To ensure that the full recognition of each particular word form in the restricted experimental context is only possible at the second syllable, we also included, in a 1:1ratio with all other stimuli, all four first syllables in isolation: [bið], [gið], [mið] and [nið]. These served as fillers to ensure identical acoustic divergence point across the four types, to be used for time-locking brain activity to, and were not analyzed as such.

The above quadruplet provided us with a way to address both lexical and semantic contrasts. By estimating the brain activity elicited by the same word-final syllable [ðə], we could compare, on the one hand, word versus meaningless pseudoword activation, putatively indicating lexical access, which we expected to find its reflection in an automatic activation of the core left temporo-frontal language system (Tyler and Marslen-Wilson, 2008). On the other hand, by comparing action versus non-action items we could address semantically-specific aspects of these activations. Previous EEG, MEG, and fMRI research has indicated automatic involvement of the brain's motor system in the comprehension of action-related verbs (Pulvermüller, 2005; Pulvermüller and Fadiga, 2010); we therefore expected more pronounced centro-frontal activity for the action verb bide, but not the concrete noun mide.

Based on the above base forms, we produced further stimuli that included a balanced morphosyntactic contrast. We took advantage of Danish morphology and the fact that the morphemes -(e)t and -(e)n can be used to express the past participle of verbs and definiteness on common nouns. This enabled us to compare the inflected items based on their syntactic congruence or incongruence, e.g., $-n$ in miden vs. *giden, and $-t$ in gidet vs. $*$ midet (where $*$ indicates a violation of the stem/affix syntactic agreement). Note that each of these pairs have identical codas $(t / n)$ that lead to grammatical/morphosytactic violation in a counterbalanced fashion: each of them is correct in combination with one but not the other stem. These were presented, in equal proportion along with the other stimuli above, to make sure syntactic properties are only recognized at the very last consonant. To 
balance for these acoustic modifications, we also included similar items based on other forms (bide[n/t] and nide[n/t], all meaningless), which were used to make a balanced design, but not analyzed as such.

The stimuli were made based on a digital recording of a male native speaker of Danish in an anechoic chamber (recording bandwitdh: $44 \mathrm{k} \mathrm{Hz}, 16$ bit, stereo). The first and second syllables of four CVCV stimuli were recorded independently, to avoid possible coarticulation effects, and cross-spliced together, such that the second syllables were physically identical across all items. The second syllable commenced at $300 \mathrm{~ms}$ after the onset of the first one, and this was the earliest time [the so-called disambiguation point (DP)] when any lexical or semantic effects could be expected in the MEG data.

To produce the morphosyntactic items, ending in [t] or [n], we cross-spliced recordings of these two morphemes onto the four main stems, to obtain words either violating or respecting rules of Danish morphology such that the exact same phonemes completed syntactic or asyntactic forms in a counterbalanced fashion. These morphemes became distinct at $408 \mathrm{~ms}$ after the word onset, and this was therefore the earliest time any morphosyntactic contrasts could affect the brain responses.

The sounds were matched for loudness, with a 1.93-dB drop between the first and the second syllables so that our stimuli sounded as natural as possible and normalized to have identical power (measured as root-mean-square, RMS). All sound editing was done using Adobe Audition CS6 software (Adobe Inc.).

In sum, the stimulus set included four CV syllables, four CVCV stems, four CVCV + [t] and four CVCV $+[\mathrm{n}]$ forms, all strictly controlled for phonological and acoustic properties. These were combined, in a pseudorandom fashion, in a single auditory sequence ensuring that the stimuli's lexical, semantic, and syntactic properties were available at stringently defined times.

\section{Procedure}

The MEG recording was conducted in an electromagnetically shielded and acoustically attenuated room (Vacuum Schmelzer GmbH). During the recordings, participants were instructed to focus on watching a silent film and to pay no attention to the sounds. The auditory stimuli were controlled using Neurobehavioral Systems Presentation v16 (Neurobehavioral Systems) and presented through in-ear-tubes (Etymotic ER-30) binaurally at $50 \mathrm{~dB}$ above individual auditory threshold.

All sixteen stimuli were presented equiprobably in a single data acquisition session intermixed in a continuous manner, with 100 pseudorandom repetitions of each stimulus resulting in 1600 epochs in total. The interstimulus onset-to-onset interval (stimulus onset asynchrony, SOA), was fixed at $1000 \mathrm{~ms}$, based on previous studies of automatic neurolinguistics processing using non-attend designs, which served as the starting point for the current paradigm. The total recording time was $28 \mathrm{~min}$.

MEG data were acquired with an Elektra Neuromag Triux MEG setup (Elekta Neuromag Oy), with 102 magnetometers and 204 planar gradiometers; for eye movement and heartbeat artifact detection two bipolar EOG and one bipolar ECG recordings were taken. Cardinal landmarks and additional head points were digitized using a Polhemus FASTRAK setup (Polhemus). Data were recorded at $1000 \mathrm{~Hz}$, a high pass filter of $0.1 \mathrm{~Hz}$ and low pass of 330 $\mathrm{Hz}$ were applied online. Head position and head movements were continuously tracked using four head position indicator coils (HPIs). The participants were lying still on a non-magnetic patient bed, with their head as close to the top of the helmet as possible, the MEG dewar being in supine position.

\section{Data preprocessing}

All data were preprocessed using MNE-python version 0.16 software package (Gramfort et al., 2013). First, continuous data were bandpass filtered from 1 to $95 \mathrm{~Hz}$, downsampled to $500 \mathrm{~Hz}$, and epoched into single-trial epochs of 1000-ms duration, starting 100 ms before and ending $900 \mathrm{~ms}$ after stimulus onset. Bad channels were automatically detected and interpolated, epochs with excessive bad channels discarded and outlier trials removed using an automatic approach (as implemented in autoreject utility; for details, see Jas et al., 2017). On average per subject there were 23.24 bad channels (median: 32, SD: 10.93) and 12.9 (SD: 18.66) bad epochs. No signal-space separation transformation (SSS; also known as maxfiltering) was applied at any stage of the preprocessing. Thereby cleaned epoch data were bandpass-filtered into five frequency bands (Dalal et al., 2011): $\alpha$ within $8-12 \mathrm{~Hz}$, $\beta$ (13-30 Hz), $\gamma$-low $(30-45 \mathrm{~Hz}), \gamma$-medium $(55-70 \mathrm{~Hz})$, and $\gamma$-high $(70-90 \mathrm{~Hz})$.

\section{Source reconstruction}

For each participant, a T1 and T2 structural MRls were obtained using a Siemens Tim Trio 3T MR scanner. The images were segmented with separate surfaces created for the gray matter, inner skull and skin using SimNIBS utility (Thielscher et al., 2015). For each subject, individual three-layer boundary element model (BEM) was calculated together with individual forward models. A common template gray matter surface was created by averaging all of the study participants, using FreeSurfer software (Dale et al., 1999).

Source reconstruction was conducted using an LCMV beamformer (Van Veen et al., 1997) using planar gradiometer data and previously developed (Westner, 2017; Westner and Dalal, 2017) Hilbert beamformer. The decision to use only gradiometers was chosen as mixing channel types is not trivial due to magnetometers and planar gradiometers producing values of different scales; furthermore planar gradiometers are less sensitive to external magnetic sources and have a better signal-tonoise ratio compared to magnetometers (Hari et al., 1988). First, for each frequency band of interest $(\alpha, \beta$, $\gamma$-low, $\gamma$-medium, and $\gamma$-high), the epochs were bandpass-filtered for each subject without subtracting the evoked signal from the single trials, as we were interested in investigating the complete information contained in the responses time locked to the auditory stimuli. Secondly, an adaptive filter was created by combining responses to all the stimuli in the paradigm, 
using a three-layer BEM and a cortically constraint source space. The adaptive filter was computed using a data covariance matrix based on all time points of the bandpass-filtered epochs; the covariance matrix was not regularized before inversion. Source orientation was optimized by using the orientation of maximum signal power (Sekihara and Nagarajan, 2008). Neural activity index (NAl; Sekihara and Nagarajan, 2008), which incorporates the weight normalization using a unit-gain beamformer, was selected as the output value. Third, after the adaptive filters were created, the single-trial epoched data were Hilbert-transformed and the adaptive filter was applied to the complex data, providing a source reconstruction of the Hilberttransformed single-trial data. Lastly, we calculated ITPC of thereby obtained single-trial source space data. This was done for each time point and in each source space location independently using the equation below:

$$
I T P C_{t f}=\left|n^{-1} \sum_{r=1}^{n} e^{i k t r}\right|
$$

where $n$ is the number of trials, $e^{i k}$ provides a complex polar representation of the phase angle $k$ on trial $r$ for the time-frequency point $t f$, where frequency is the frequency band (for review, see Cohen, 2014, chapter 19, especially pp. 244-245). This resulted in a single ITPC time course for each point in the source space for each subject.

After the source space ITPC data were calculated for each subject, the individual data were morphed onto a common template surface (5124 vertices) created as the mean over all individual participants for data standardization across the group. Finally, the data were smoothed with a 10-ms rolling window mean in temporal dimension for each source independently.

\section{MVPA}

For each participant, the morphed ITPC time series for each point in the source space was extracted based on the contrast in question. Common for all the MVPA was the classification over time, i.e., for each time point a classifier pipeline was applied giving a classification score over time. We used the entire cortical source space for the classification at each time point. As it was comprised of 5124 vertices, it gave 5124 features per time point.

The classifier pipeline was constructed in MNE-python using scikit-learn utility (Pedregosa et al., 2011) and composed of three steps. First, the features were standardized (z-scored); the standardization was done across all vertices in the source space at each time point independently using the training set and then applied to the test set. Second, feature selection was done using crossvalidated Lasso model (stratified folds, $n=4$ ) to create a sparse feature space that was adaptive for each time point, i.e., allowing for number of relevant features to be different for different time points. Lastly, a logistic regression $(C=1)$ was used to classify the two contrasts, and receiver operating characteristic area under the curve (ROC-AUC) was used as the classification score. The pipeline was applied across subjects, meaning that we obtained a decoding score over time for all participants and, hence, we only looked for effects that could hold across the subjects in the tested population.

MVPA can easily overfit data (i.e., become biased toward a specific response) and, to prevent that from happening, cross-validation was used for the pipeline. Simply put, cross-validation makes use of all the data by first splitting the data into two smaller data sets. One set, called the training set, is used for standardizing the features and fitting (training) the MVPA model. The other data set, called the test set, is then used to test accuracy of the fitted model (i.e., trained model) by trying to predict the class of the new label and, by comparing the prediction to the actual class of the test set, we can calculate the ROC-AUC for the model. By creating new training and test sets from the full data set, it is possible to use all the data for both training and testing (for more details and strategies for cross-validating brain imaging data, see Varoquaux et al., 2017).

All the steps in the pipeline were cross-validated with stratified folds $(n=5)$. Stratified folds imply that ratio of classes in the all the data are maintained in all the crossvalidated folds; e.g., in the lexical condition there are three real words and one pseudoword for each participant, so in the stratified folds the ratio $3: 1$ would remain such that even when shuffled there will always be a 3:1 ratio. For the semantic condition and morphosyntax comparison the ratio was $1: 1$, i.e., $50 \%$. We had 17 participants in the study and it was, therefore, impossible to have an equal number in each of the cross-validation folds and to make cross-validation splits that have the exact same number of participants in all folds and keep the ratio the same. However, by using stratified folds we kept the balance between the folds as equal as possible.

To test for statistical significance of the classification we used permutation tests (Ojala and Garriga, 2010). A permutation test was performed for each time point independently. First, the arrangement of the labels was shuffled, e.g., action verb and object noun in the semantic condition, such that the data might be from an action verb but the label tell the MVPA algorithm that it is an object noun. By repeatedly shuffling the labels and running the classification ( $n=2000)$, we can build a null distribution of random ROC-AUC scores. This null distribution is interpreted as the distribution of what the ROC-AUC score could be just by chance. Hence, we can then assess the classification score we had from the real labels by comparing to the null distribution. If number of random scores that are better than the actual classification score is less than or equal $5 \%$, the classification score is said to be better than chance, where $5 \%$ is the $\alpha$ level chosen.

To optimize the computational time, only classification scores that surpassed the threshold (calculated, for each frequency band independently, as the mean score of the baseline plus 1.5 SDs of the baseline) were tested for statistical significance. So, for each time point where the ROC-AUC score was above the threshold we made a permutation test $(n=2000)$. As the minimum $p$ value that can be obtained with a permutation test is dependent on 
Table 1. Table of significant clusters in the lexical condition sorted by time from the divergence point

\begin{tabular}{|c|c|c|c|c|c|c|c|c|}
\hline \multicolumn{9}{|c|}{ Lexical } \\
\hline Band & Peak (\%) & $\begin{array}{l}\text { Peak } \\
\text { SD (\%) }\end{array}$ & $\begin{array}{c}\text { Peak } \\
\text { time }(\mathrm{ms})\end{array}$ & $\begin{array}{l}\text { Cluster } \\
\text { start (ms) }\end{array}$ & $\begin{array}{l}\text { Cluster } \\
\text { end (ms) }\end{array}$ & $\begin{array}{c}\text { Cluster } \\
\text { length (ms) }\end{array}$ & $\begin{array}{l}\text { Cluster } \\
\text { mean (\%) }\end{array}$ & $\begin{array}{l}\text { Cluster } \\
\text { SD (\%) }\end{array}$ \\
\hline$\gamma$-Medium & 88.53 & 6.43 & 66 & 62 & 76 & 14 & 83.20 & 4.57 \\
\hline$\gamma$-High & 87.88 & 9.29 & 358 & 350 & 366 & 16 & 80.19 & 4.95 \\
\hline$\gamma$-Low & 87.65 & 11.08 & 440 & 432 & 448 & 16 & 81.34 & 4.99 \\
\hline$\gamma$-Low & 87.71 & 8.83 & 516 & 510 & 534 & 24 & 81.34 & 4.36 \\
\hline
\end{tabular}

Peak is highest ROC-AUC scores of the cluster. Peak SD is the standard deviation (SD) of cross-validation folds for the peak ROC-AUC score. Peak time is the time of the peak from DP. Cluster start is the start time of the cluster from DP. Cluster end is the end time of the cluster from DP. Cluster length is the length of the cluster. Cluster mean is the mean ROC-AUC score of the cluster. Cluster SD is the SD of the cluster mean across cross-validation folds.

the number of permutations run, the $p$ value is defined as $p_{\mathrm{u}}=(b+1) /(m+1)$, where $b$ is the number of times the permutation was equal or more extreme then the observed classification value and $m$ is the number of permutations, and 1 is added to $b$ and to $m$ to ensure that the estimated $p$ value is not zero and to avoid division by zero (Phipson and Smyth, 2010; Puolivali et al., 2018). So, the $p$ value in a permutation test does not behave in the same way as a $p$ values in a parametric test, it is rather a description of observed probability that the given classifier is better than chance. Since we perform a series of permutations, i.e., one for each time point, we need to consider the multiple comparison problem, i.e., the problem that some tests will be significant purely by chance and not be a true effect. We opted to apply cluster threshold correction, i.e., there needs to be a continuous range of significant time points (at least $10 \mathrm{~ms}$ or longer) to accept it as a non-random effect. The 10-ms threshold is based on previous language and auditory research $(\mathrm{Ha}-$ goort and Brown, 2000; Wang et al., 2012; Edmonds and Krumbholz, 2014), typically using $10 \mathrm{~ms}$ as the shortest bin duration to test. It should be noted that only two of the thirteen clusters reported as significant at $10 \mathrm{~ms}$, the rest are longer, ranging from 12 to $42 \mathrm{~ms}$.

For each linguistic contrast we fitted the classification pipeline independently. For the lexical contrast, we tested whether we could classify lexical features, i.e., if the participant heard a real word versus an acoustically similar pseudoword (see above, Stimuli). In the semantic contrast, we tested classification of the action verb versus object noun. In the syntactic contrast, we tested whether we could correctly classify ungrammatical versus grammatically correct items (irrespective of their acoustic features, i.e., *midet, *giden vs miden, gidet). Each contrast was tested from the relevant DP (300 ms for lexis and semantics and $408 \mathrm{~ms}$ for morphosyntax) through the end of the epoch. Latencies reported in Results below are measured relative to DP.

\section{Results}

\section{Lexical contrast}

For the lexical contrast (Figs. 2-4; Table 1), we found that it was possible to significantly decode words versus pseudowords in the $\beta, \gamma$-low, $\gamma$-medium, and $\gamma$-high bands. The earliest significant decoding was achieved in the $\gamma$-medium band already at $62-76 \mathrm{~ms}$ after the DP. The pattern of classifier-selected features in the left hemisphere included the frontal lobe (BA 44, border of BA 6/BA 8, BA 4), parietal BA 7, junction of BA 7/39, and BA $39 / 19$. In the right hemisphere, features in temporal lobe BA 22 and frontal BA 9, BA4 were selected. The highest ROC-AUC score was in the $\gamma$-low band at $224 \mathrm{~ms}$ after DP (ROC-AUC: $94.35 \%$, SD: $4.5 \%$ ). The cluster including this AUC-ROC peak spanned from 222 to $238 \mathrm{~ms}$ and involved a broad pattern of features including, in the left hemisphere, temporal lobe (BA 22), frontal areas (BA 11, border of BA10/47, BA44 dorsal/posterior), and parietal areas (BA 40, BA 7 as well as BA 3/1/2). In the right hemisphere, it included the temporal lobe (BA 22, BA 23, BA 43), frontal areas (BA 11, border of BA 9/10/46, posterior dorsal BA44), and parietal areas (BA 39, BA 40, BA 7 , BA 1/2). No significant classification results were obtained in the $\alpha$ band.

\section{Semantic contrast}

In the semantic contrast (Figs. 2, 5, 6; Table 2), the peak classification score was found in the $\alpha$ band at $106 \mathrm{~ms}$ after DP (ROC-AUC: 91.11\%, SD: 12.96\%). The cluster including the peak ROC-AUC was between 80 and 122 ms after the onset of the second syllable disambiguating the semantics of the particular form. The cluster comprised a pattern of features including both temporal (BA 22) and frontal areas (BA 9, BA 44, BA 45, BA46) of the left hemisphere. In the right hemisphere, in addition to the temporal (BA 21, BA 22, BA 38, BA 42) and the frontal lobe (BA 6, BA 9, BA 11, BA 44), it also involved parietal areas (BA 7 and BA 2).

\section{(Morpho)syntactic contrast}

The results of MVPA classification of grammatically correct versus incorrect inflections (Figs. 2, 7, 8; Table 3) showed the peak ROC-AUC score in the $\gamma$-low band at 90 ms after the syntactic DP (ROC-AUC: 69.22\%, SD: $13.05 \%)$. The significant classification cluster on the left hemisphere included the temporal lobe (BA 21, BA 22, BA 37, BA 41), frontal areas (BA 4, BA 9 BA 10, BA 11), as well as parietal areas (BA 40/7/2), and occipital area BA 19. In the right hemisphere, it involved temporal (BA 21, 22, 38, 42), frontal (BA 44, 11, 4, 6, 9), parietal (BA 39, BA 40, 2, $1,7)$, and occipital (BA 18, 9) areas. 

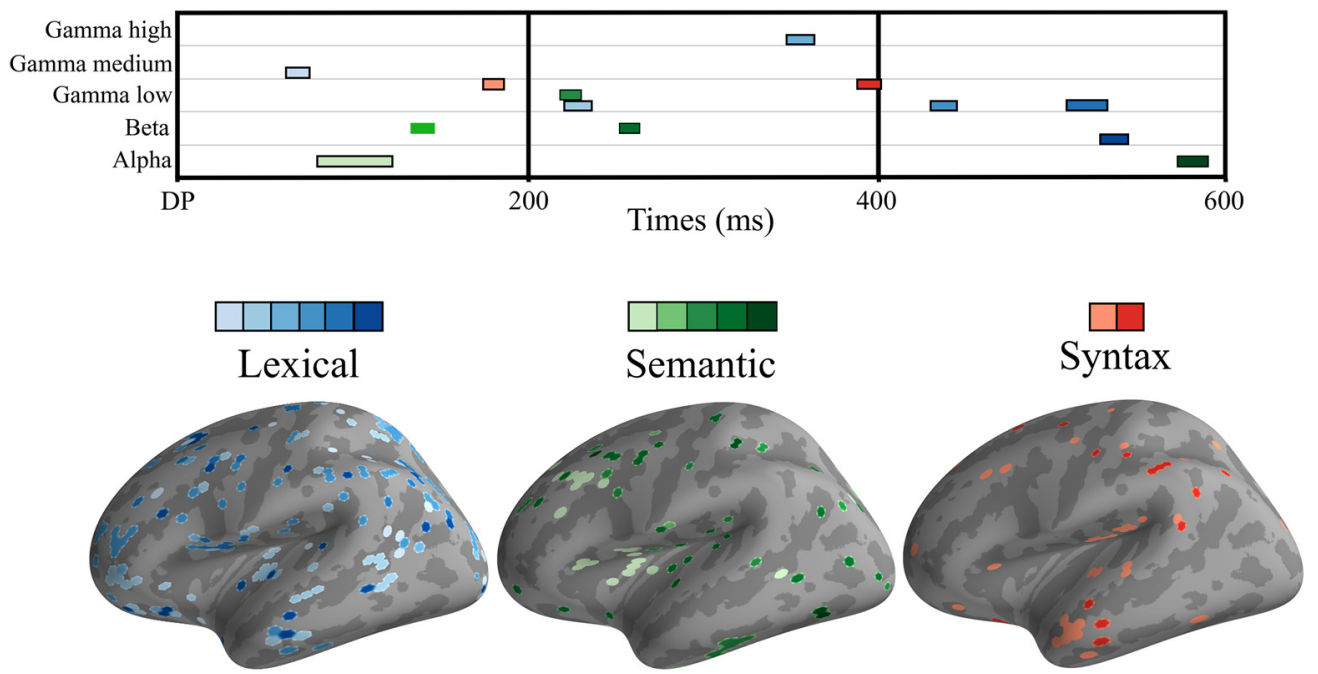

Figure 2. Top, Heatmap of significant clusters across three linguistic contrasts, five frequency bands, and time. Lexical condition in blue colors, semantic in green, and syntax in red. Bottom, Surface topography of significant effects. For all conditions, colors go from lighter to darker as latency becomes longer.

\section{Classification of amplitude data}

We also tested ad hoc whether we could decode language properties using amplitude data. We only found significant clusters in semantic in the $\gamma$-medium band from 500 to $512 \mathrm{~ms}$ after DP. For the morphosyntactic condition, we found that we could classify correctly in the $\gamma$-high bands (from 114 to $128 \mathrm{~ms}$ after DP) and $\beta$ band (from 248 to $262 \mathrm{~ms}$ after DP). As we were not able to decode all conditions successfully, we have left out the amplitude results from further discussion.

\section{Discussion}

In this study, we suggested and tested a paradigm which, in the absence of focused attention on the auditory input or any explicit stimulus-focused behavioral task, addressed different levels of neurolinguistic information processing in the brain using a carefully crafted set of spoken stimuli with strictly controlled acoustic/psycholinguistic contrasts. We registered the brain's activity elicited by these speech stimuli using high-density wholehead MEG set-up, and analyzed it using machine learning-based MVPA techniques applied to inter-rial phase coherence in a range of frequency bands, at the level of cortical sources calculated using individual MRIs. The results indicated that, by using this approach, we were able to successfully classify lexical, semantic, and morphosyntactic contrasts (for an overview of results, see Table 4). These effects were exhibited in different frequency bands and at different times. Below, we will briefly discuss these results in more detail.

\section{Lexical contrast}

In the lexical contrast, we found clusters of significant decoding performance (with a peak classification score of $\sim 94 \%$ ) over the entire duration of the analysis epoch starting from $\sim 60 \mathrm{~ms}$ after the divergence point. These predominantly occurred in the $\gamma$ range (including all three sub-bands tested) and were underpinned by activity in bilateral temporo-parietal and frontal clusters. This early onset of lexical effects here is in line with previous ERF and ERP studies that used similar non-attend auditory designs (Pulvermüller and Shtyrov, 2006; MacGregor et al., 2012; Shtyrov and Lenzen, 2017) and showed that the brain responds differently to meaningful words versus meaningless pseudoword stimuli from $\sim 50$ to $80 \mathrm{~ms}$ after the acoustic input allows to identify the lexicality of stimuli, with additional lexicality-driven activity spanning across peaks until $\sim 400 \mathrm{~ms}$. Such ERP/ERF results (Shtyrov et al., 2010; see also Shtyrov et al., 2005) have reported similar perisylvian configuration of bilateral source activations; importantly, here we find them for higher-frequency phase coherence values, not reported previously.

Increased activity in the $\gamma$ band (quantified as spectral power or as event-related synchronization) has previously been linked to lexical access possibly underpinning synchronization of neural elements making up distributed word memory circuits (Lutzenberger et al., 1994; Pulvermüller et al., 1996; see also Tavabi et al., 2011). What our findings suggest is that this process may involve multiple synchronization steps expressed as phase resetting (an important mechanisms in information processing; Canavier, 2015) at different times, frequencies and neuroanatomical locations.

These previous studies have typically found a single power peak in low $\gamma$ dynamics, whereas we here find a series of activations at frequencies up to $90 \mathrm{~Hz}$, potentially reflecting the specific advantages of machine learning techniques in classifying distributed clusters of activity. We also found a single significant $\beta$ cluster at the end of the epoch ( $>500 \mathrm{~ms}$ post-DP). This is fully in line with previous research highlighting the role of $\beta$ oscillations in lexicosemantic storage and processing (Brennan et al., 2014; Bakker et al., 2015), although our data suggest that this $\beta$ activity is secondary in relation to the almost immediate phase resetting in the $\gamma$ band. Note that while a number of above studies have also identified $\theta$ band 
A Model patterns: real words vs pseudo word
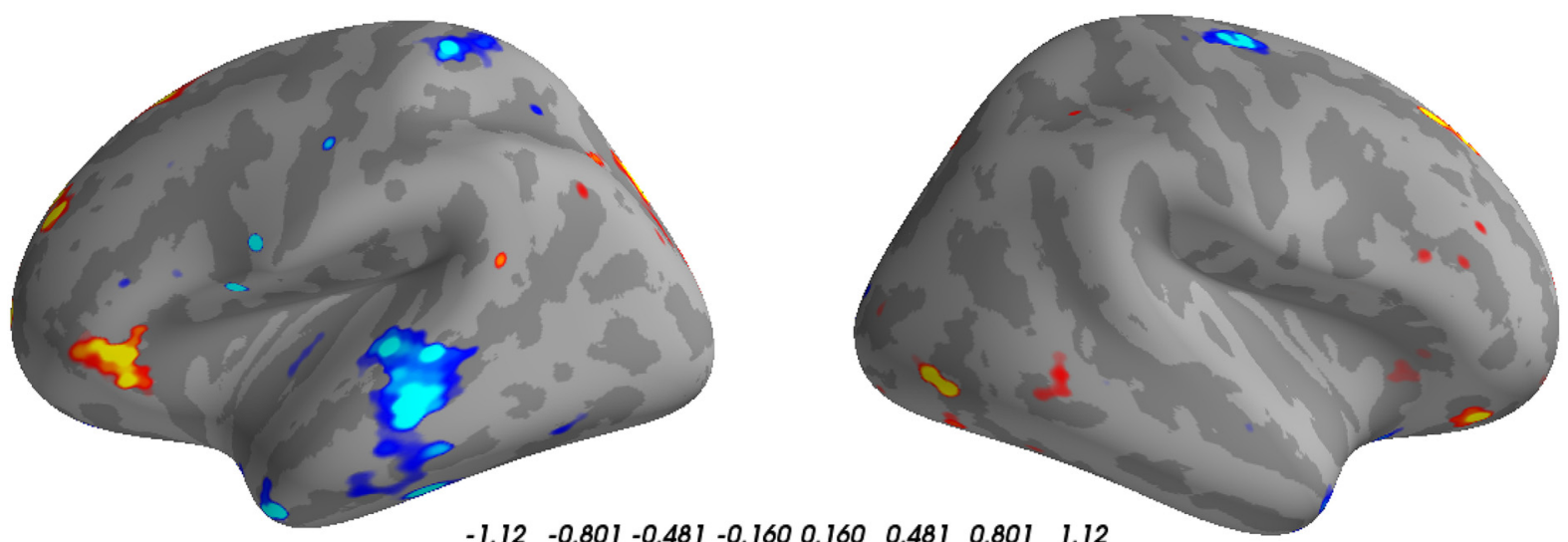

$\begin{array}{llllllllll}-1.12 & -0.801 & -0.481 & -0.160 & 0.160 & 0.481 & 0.801 & 1.12\end{array}$

Real words (zscored ITPC) Pseudo word

\section{B ITPC contrast between real words and pseudo word}

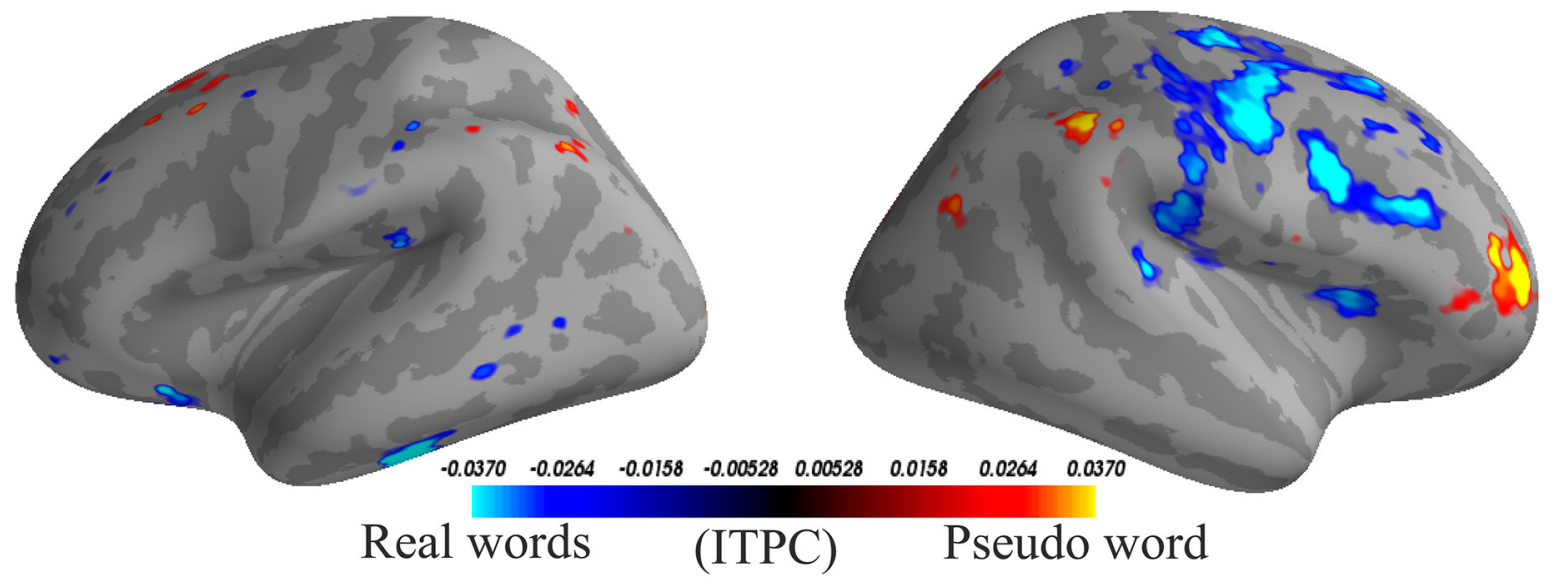

c Time course of average ITPC
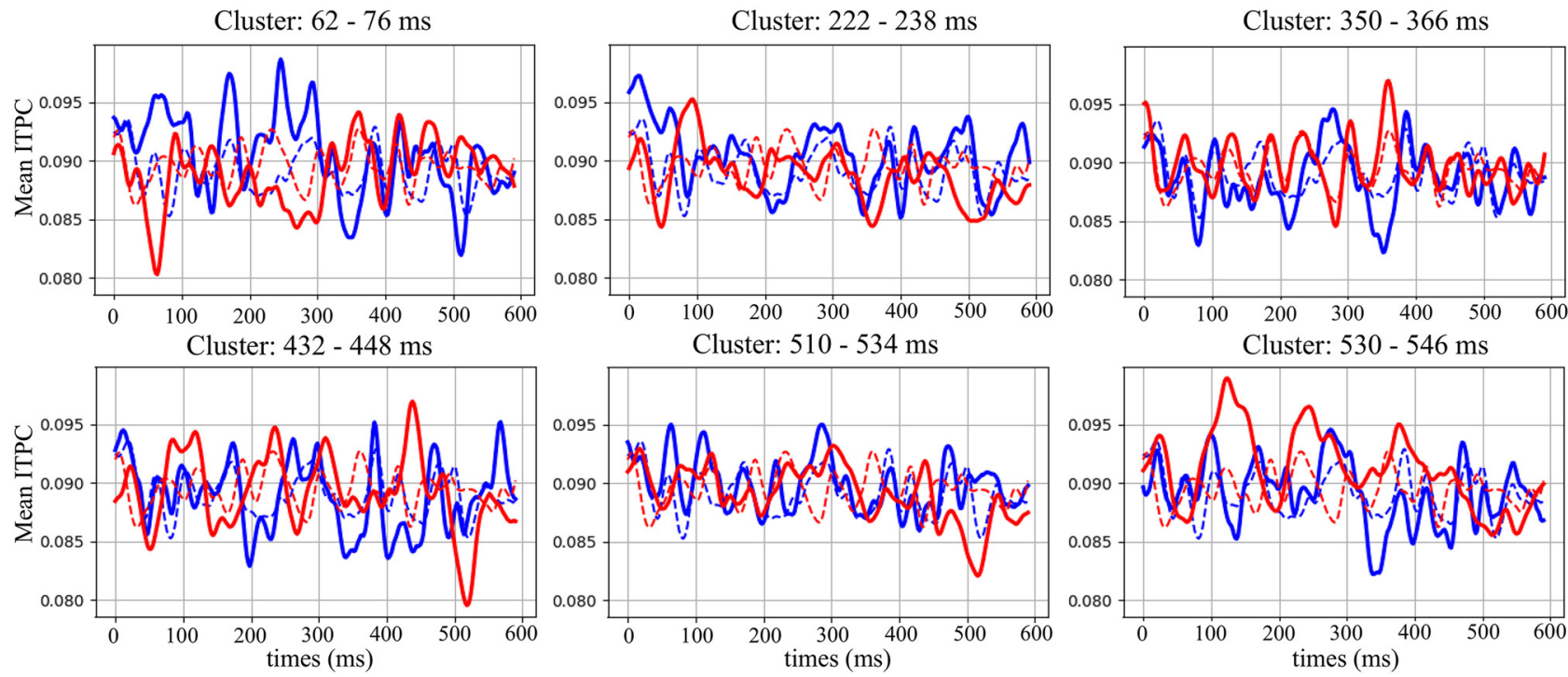

Figure 3. A, Model patterns: interpreting coefficients of a machine learning model is not trivial and a high coefficient value does not 


\section{continued}

necessitate a high signal value in the MEG data (for details, see Haufe et al., 2014). "Model patterns" are a way to highlight the signal in a neurophysiological sensible way that is directly interpretable compared to the raw coefficients (Haufe et al., 2014). We show top and bottom 5\% of the patterns in the $\gamma$-low band from 222 to $238 \mathrm{~ms}$. Blue colors are areas of activation able to predict real words and yellow/red are areas used to predict pseudo word. B. Average top and bottom $5 \%$ of ITPC difference; blue colors indicate higher ITPC for real words and yellow/red colors indicate higher ITPC for pseudo word $\gamma$-low band from 222 to 238 ms. $\boldsymbol{C}$, Average ITPC over time; solid lines are the average of the selected features, dashed lines are the average of all vertices in the source space. Time 0 is the divergence point, when stimuli could be recognized from the available acoustic information.

activity in relation to some aspects of speech processing (e.g., syllable tracking; Luo and Poeppel, 2007, 2012), we optimized our recording for time (with a view of potential applied use) that led to short baselines not suitable for $\theta$ analysis. There is, however, compelling evidence of a relation between $\theta$ and $\gamma$ band activity (Canolty et al., 2006), and future research using similar paradigms could investigate whether the current ITPC findings may also have a counterpart in the $\theta$ range.

\section{Semantic contrast}

The semantic contrast between the action verb and non-action noun has indicated activity from $\sim 100 \mathrm{~ms}$ after the divergence point in $\alpha, \beta$ and, to a smaller degree, in a lower $\gamma$ range, with peak classification scores of $\sim 91$, 75 and $85 \%$, respectively. This mostly involved temporofrontal cortices, largely overlapping with the core language systems, but importantly, indicated elevated frontal involvement, including clusters of activity in inferiorfrontal (BA44, 45) and motor (BA6) areas, which is compatible with the motor system involvement in action word processing, posited previously (Pulvermüller et al., 2006). Previous EEG and MEG research into the brain basis of action-related semantics has found ERF and ERP correlates between 80 and $200 \mathrm{~ms}$, indicating near-immediate and largely automated activation of the motor strip in action word comprehension (Shtyrov et al., 2004, 2014). In line with this previous research, the features indicated here by the semantic contrast did not include many in the temporal lobe but mostly in the inferior-frontal and prefrontal areas in the left and right hemispheres. While the timing of this activation was generally similar, and thus largely parallel, to the lexical processes above, the spectral composition was different, with significant ITPC findings in a lower frequency range. Changes in $\alpha$ and especially $\beta$ power have previously been reported as related to single word semantics, including action word semantics in particular (Vukovic and Shtyrov, 2014; Bakker et al., 2015). What we show here is that the phase resetting likely linked to these changes can reliably classify words with different meaning. In oscillatory space our results are in line with those previously reported by Mamashli et al. (2019) who investigated functional connectivity across regions of interest and found activity in the $\alpha$, $\beta$ and low $\gamma$ bands, while Haarmann and Cameron (2005) reported a change of coherence in the $10-$ to $14-\mathrm{Hz}$ range when retaining a sentence.

\section{Syntax contrast}

Successful classification of the morphosyntactic contrast was achieved in the $\gamma$-low range exclusively and was found to commence at $\sim 100 \mathrm{~ms}$ after the syntactic divergence point. While, in terms of the absolute stimulus timing, this difference was later than the lexical and semantic findings above, it is important to note the syntactic disambiguation in the stimulus itself was also possible at a later time, as it was determined by the final consonant $(n / t)$. Thus, in terms of the relative timing, the syntactic properties appear to be assessed roughly in parallel to other tested features, overall in line with the view positing near-simultaneous onset of neurolinguistics processing of different information types (Marslen-Wilson, 1987; Ha-

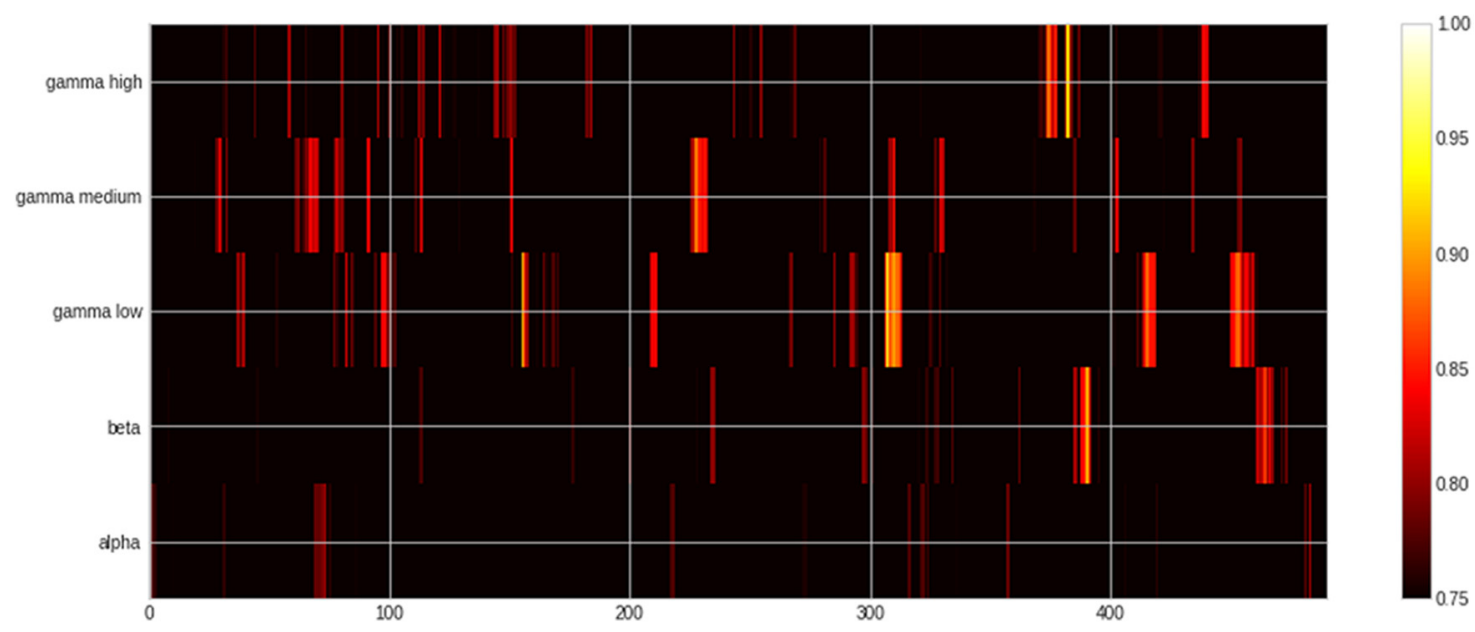

Figure 4. Heatmap of ROC-AUC scores for all bands in lexical condition. Note that chance in this condition is $75 \%$. Time is relative to DP. 
A Model patterns: action verb vs object noun

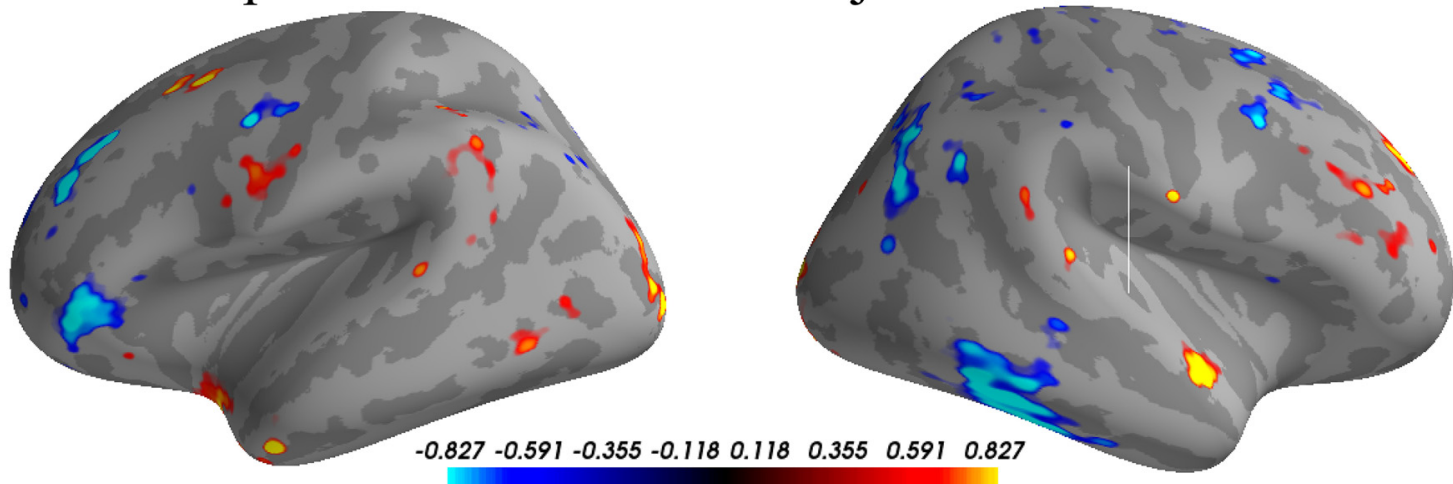

Action verb (zscored ITPC) Object noun

\section{B ITPC contrast between action verb vs object noun}
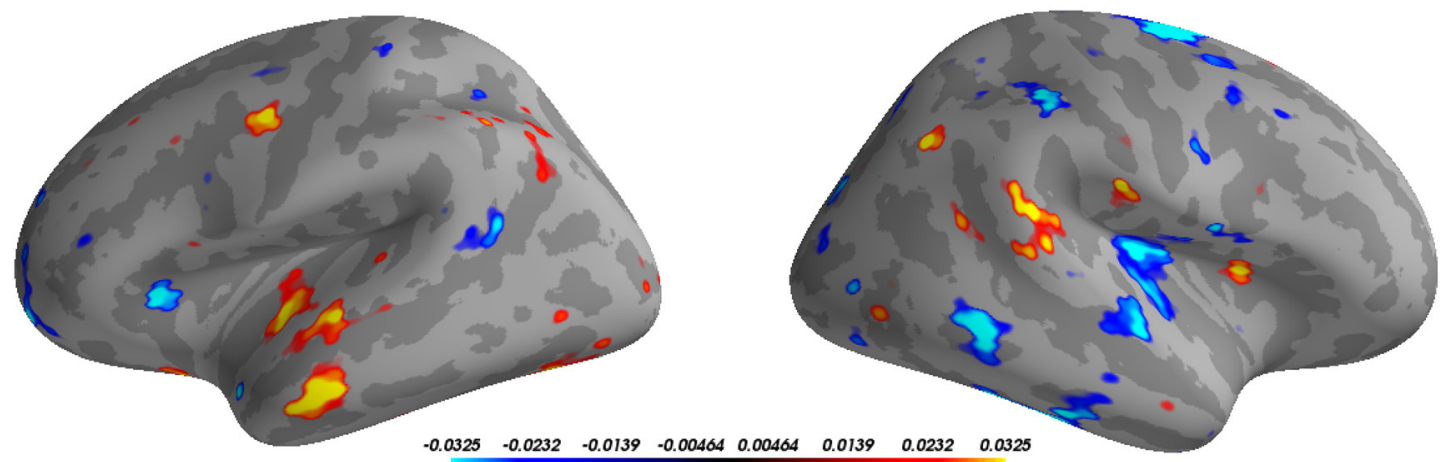

Action verb

(ITPC)

Object noun

c Time course of average ITPC
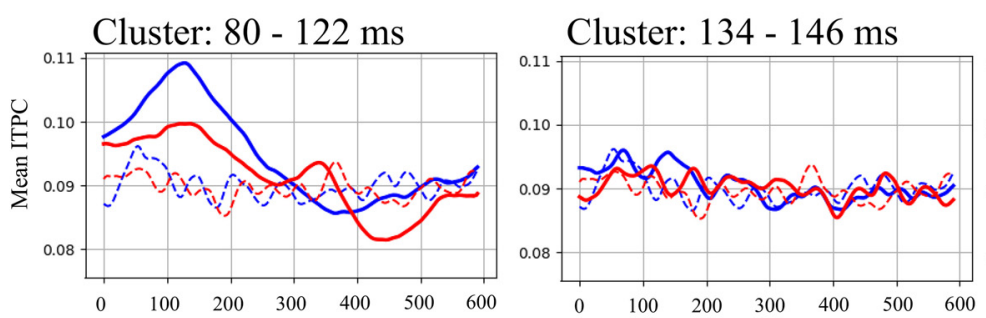

Cluster: $220-230 \mathrm{~ms}$
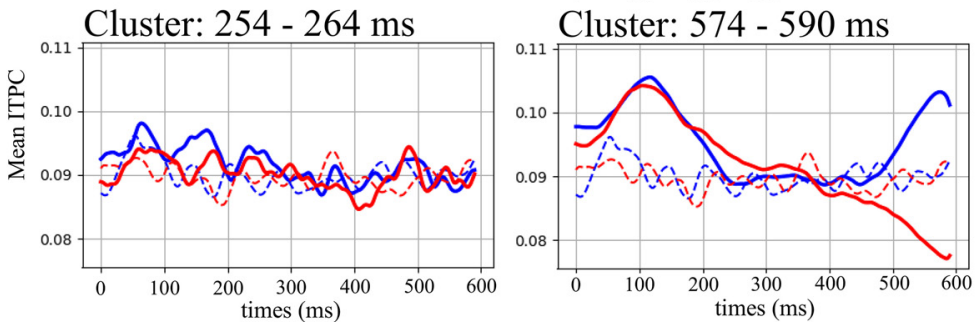

Mean selected features for action verb I I Mean of all features for action verb
Mean selected features for object noun I I Mean of all features for object noun
Significant cluster

Figure 5. A, Model patterns (see also Fig. 4 legend): top and bottom 5\% of the patterns in the the $\alpha$ band from 80 to 122 ms. Blue colors are areas used to predict action verb and yellow/red are areas used to predict object noun. $\boldsymbol{B}$, Average top and bottom $5 \%$ of ITPC difference, blue colors indicating higher ITPC for action verb and yellow/red indicating higher ITPC for object noun from 80 
continued

to $122 \mathrm{~ms}$. C, Average ITPC over time, solid lines are the average of the selected features, dashed lines are the average of all vertices in the source space. Time 0 is the divergence point, when stimuli could be recognized from the available acoustic information.

goort, 2004). The timing is in line with the findings of ELAN literature that suggested syntactic parsing to sometimes start as early as $50 \mathrm{~ms}$ in an automatic fashion (Herrmann et al., 2011). Findings of links between $\gamma$ band activity and syntactic parsing have been reported in the literature previously (Lewis et al., 2015), including morphosyntactic processing of the kind broadly similar to that required in comprehension of complex words used here (Levy et al., 2014). Importantly, we used a fully balanced set of contrasts, with highly matched word stimuli in which morphosyntactic (in)correctness was carried by physically the same phonemes, i.e., [n] and [t] equally employed in both sets. This activation was underpinned by broad temporofrontal networks in both hemispheres, in line with previous literature (Hickok and Poeppel, 2007; Friederici, 2011; Bozic et al., 2015). We have also found activity in parietal and occipital areas, not commonly reported in syntax studies and therefore requiring validation in future research; notably, the values here do not reflect absolute activity (e.g., activity) as such but rather reliable classification of activation, however small it may be. Some studies investigating oscillatory neural activity in relation to syntactic processing have found that there is a link between $\alpha$ power and syntactic properties of phrases (Leiberg et al., 2006). While we do not find any similar activity in the $\alpha$ range for our morphosyntactic condition, that may be due to the use of single word stimuli with morphosyntactic modifications (rather than phrases with more elaborate syntax) in our paradigm.

Of the three linguistic contrasts tested, the morphosyntactic one had the lowest decoding percentage (peak at $\sim 69 \%$ ). One possible reason for this is that it used two acoustically different stimuli, words ending in [n] and [t], with the grammatical correctness independent of the word ending. The different acoustic properties, later onset of [t] than [n] and different amplitude envelopes of the two, may have smeared the effect in time leading to poorer (but nevertheless significant) classification results. Future studies could use other contrasts and different languages to improve classification results.

\section{MVPA of MEG ITPC data as a tool for objective assessment of neurolinguistics processes}

Previous literature has shown that passive paradigms can be used to investigate language processing (Shtyrov et al., 2012; Gansonre et al., 2018), an important first step toward assessing participants and patient groups that have difficulties responding verbally or in other behavioral ways. However, the next necessary step is to optimize the analysis of data obtained in such paradigms. Important

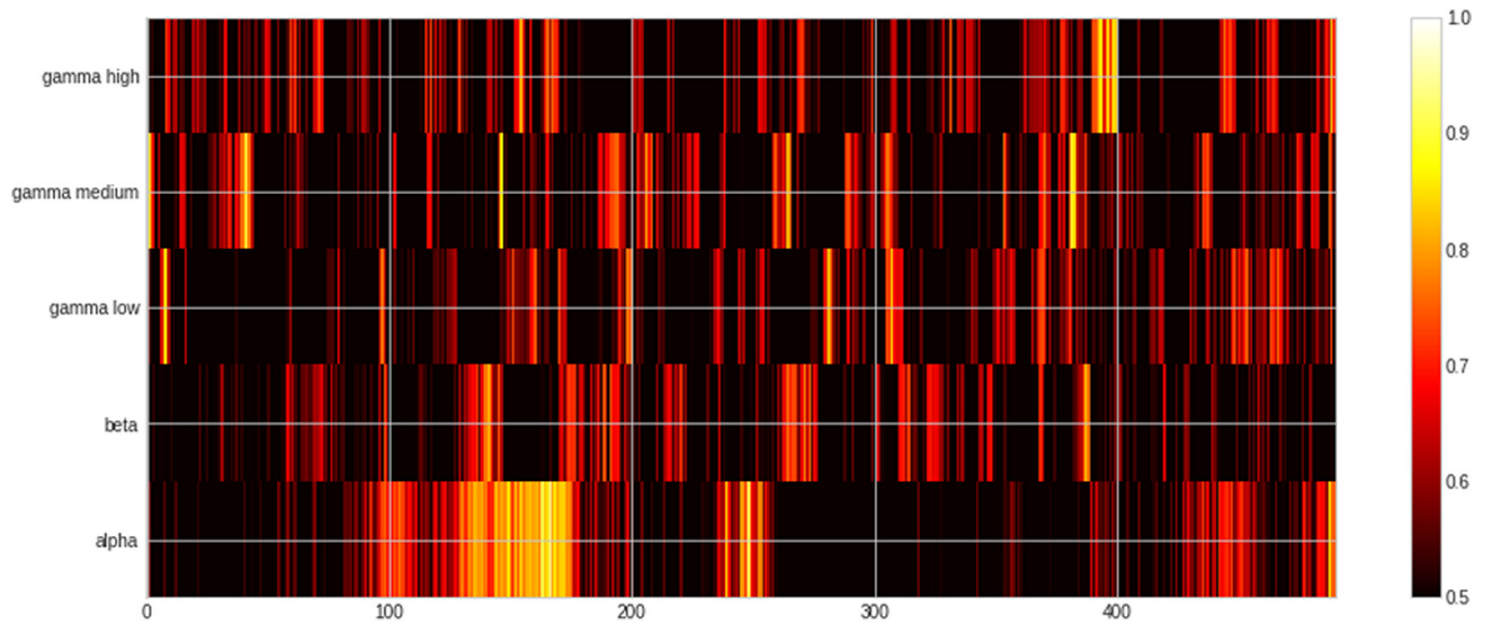

Figure 6. Heatmap of ROC-AUC scores for all bands in semantic condition.

Table 2. Table of significant clusters in the semantic condition sorted by time from the divergence point

\begin{tabular}{|c|c|c|c|c|c|c|c|c|}
\hline \multicolumn{9}{|c|}{ Semantics } \\
\hline & & Peak & Peak & Cluster & Cluster & Cluster & Cluster & Cluster \\
\hline Band & Peak (\%) & SD (\%) & time (ms) & start (ms) & end (ms) & length (ms) & mean (\%) & SD (\%) \\
\hline$\alpha$ & 91.11 & 12.96 & 106 & 80 & 122 & 42 & 68.21 & 9.77 \\
\hline$\beta$ & 75.00 & 19.08 & 138 & 134 & 146 & 12 & 69.62 & 5.25 \\
\hline$\gamma$-Low & 84.58 & 9.01 & 224 & 220 & 230 & 10 & 71.85 & 7.06 \\
\hline$\beta$ & 70.00 & 13.43 & 256 & 254 & 264 & 10 & 66.53 & 3.37 \\
\hline$\alpha$ & 85.83 & 3.74 & 584 & 574 & 590 & 16 & 71.71 & 7.62 \\
\hline
\end{tabular}

See the legend of Table 1 for an explanation of the columns. 


\section{A Model patterns of activity related to predicting correct and incorrect syntax}

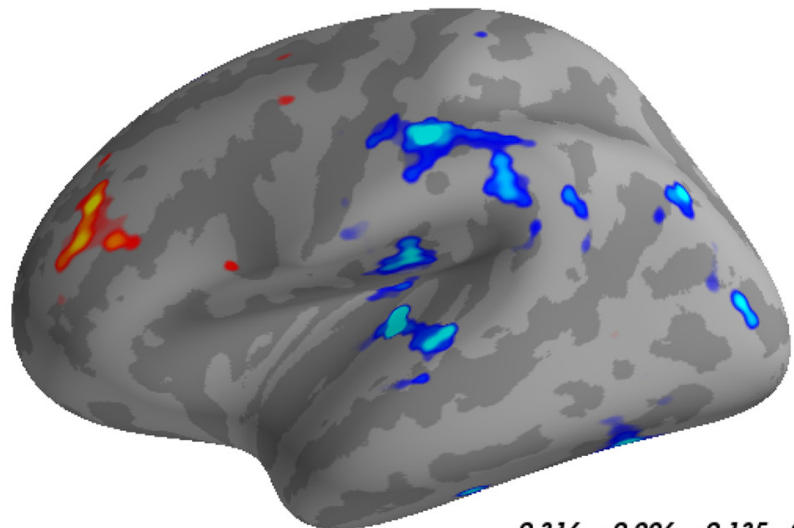

$\begin{array}{llllllll}-0.316 & -0.226 & -0.135 & -0.0451 & 0.0451 & 0.135 & 0.226 & 0.316\end{array}$

correct syntax

(zscored ITPC) incorrect syntax

\section{B ITPC contrast of correct and incorrect syntax}
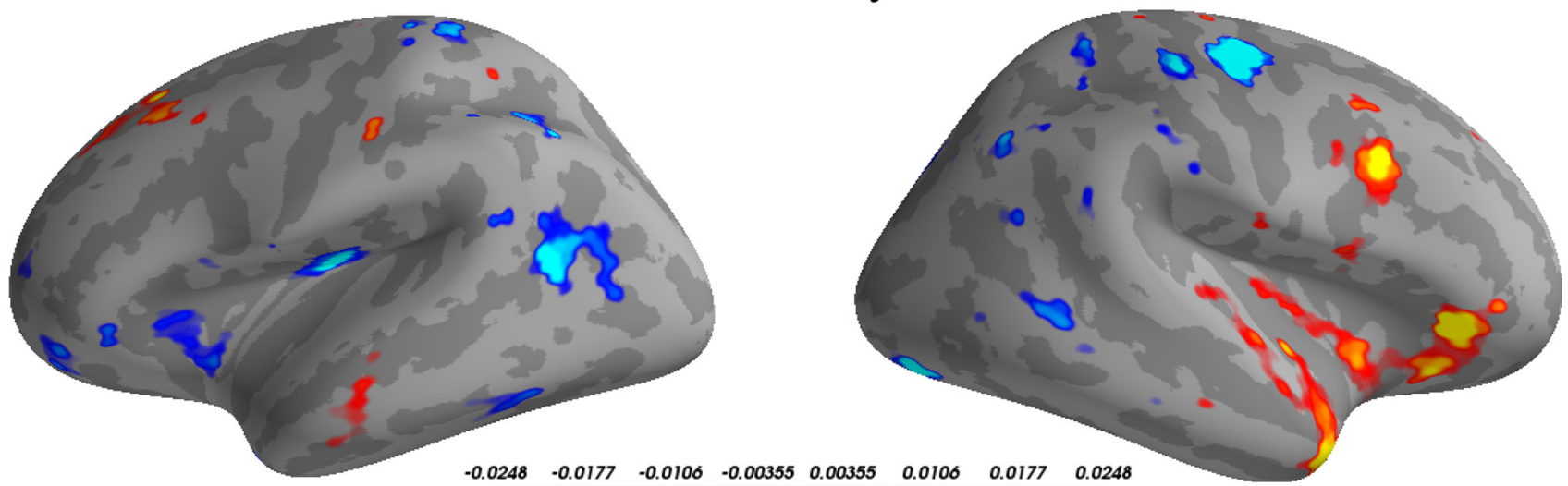

correct syntax

(ITPC)

incorrect syntax

\section{Time course of average ITPC}
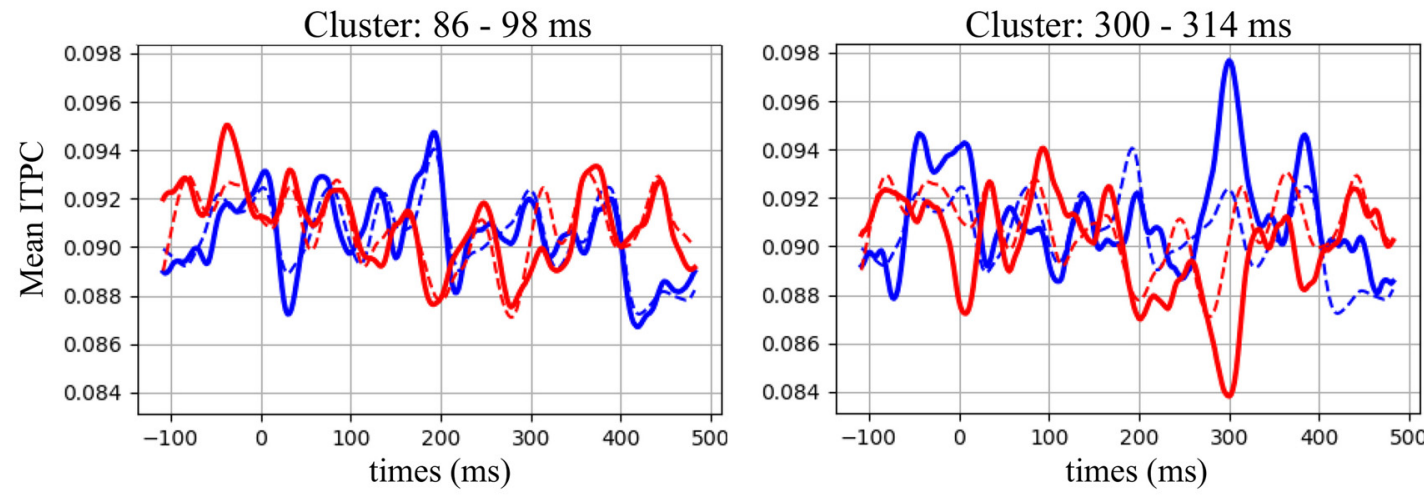

Mean selected features for correct syntax

- - Mean of all features for correct syntax

Mean selected features for incorrect syntax

- - Mean of all features for incorrect syntax

Figure 7. A, Model patterns: top and bottom $5 \%$ of the patterns in the $\gamma$-low band from 84 to 98 ms. Blue colors are areas used to predict correct syntax and yellow/red are areas used to predict incorrect syntax. $\boldsymbol{B}$, Average top and bottom $5 \%$ of ITPC difference. Blue colors indicate higher ITPC for correct syntax and yellow/red colors indicate higher ITPC $\gamma$-low band from 84 to 98 ms. $\mathbf{C}$, Average ITPC over time; solid lines are the average of the selected features, dashed lines are the average of all vertices in the source space. Time is relative to the divergence point. 


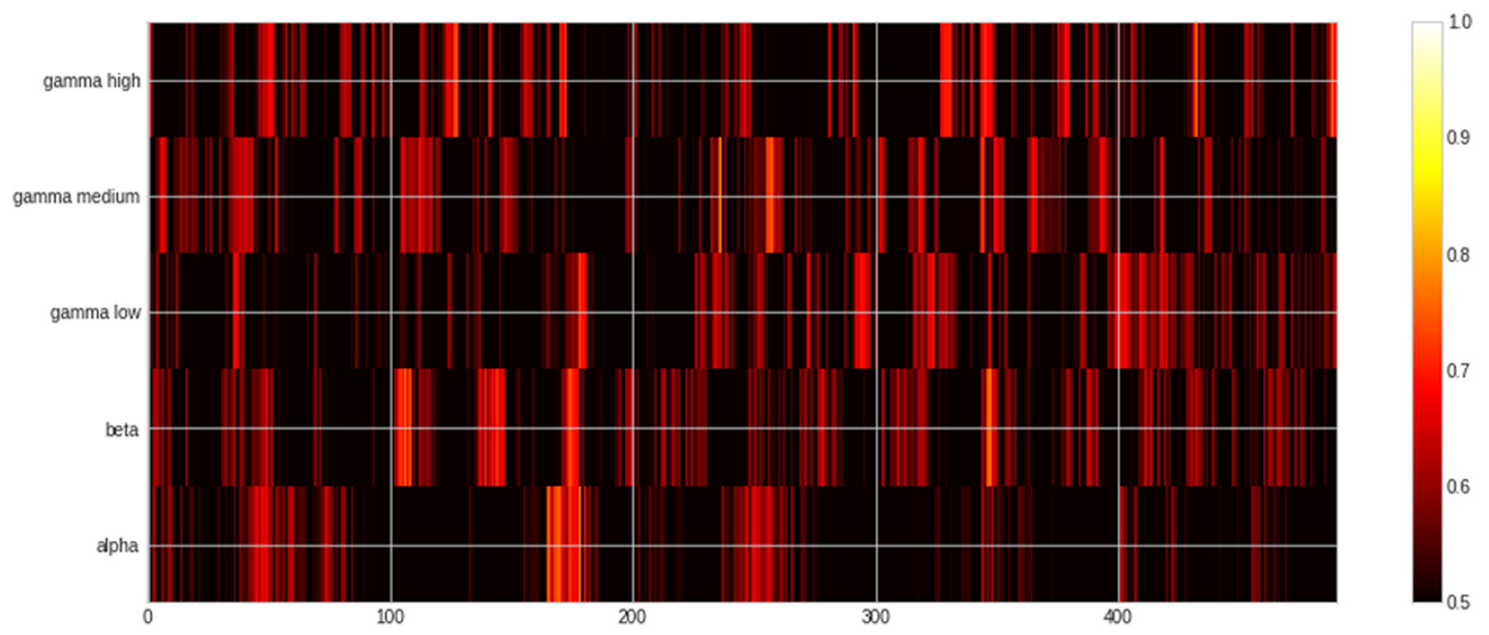

Figure 8. Heatmap of ROC-AUC scores for all bands in syntax condition.

issues arise when trying to automate MEG analysis both at preprocessing stage and at the level of identifying significant effects and differences either within or between groups. What we present here is a tentative proposal to solve these issues. By using the automated data cleanup protocol combined with a single-trial beamformer reconstruction, we reduce the number of manual steps needed; and further, by applying MVPA we avoid having to a priori select time and regions of interest (ROIs) for the statistical assessment of the difference between groups. The focus on oscillatory dynamics and ITPC provides us the ability to assess activity in different frequency bands simultaneously, resulting in a more detailed picture of the neural activity related to neurolinguistic processing. Previously, such analyses have been hampered by the number of tests that are needed to statistically evaluate the differences across groups/conditions. To solve this problem, we used MVPA, as this approach makes use of the powerful machine learning techniques capable of assess- ing the effects statistically in an unbiased fashion. So, by combining ITPC and MVPA we have the possibility to assess data in a detailed and yet exploratory manner.

Common to the effects across all conditions is that they last a relatively short time compared to the length of the trials. It is worth remembering that we are looking for clusters of time where we can decode a difference in the signals that are compared, which may help explain temporally sparse results. It is also worth noting that one cannot from a significant cluster alone conclude what exact processes underpin it. However, comparing the ITPC data and the patterns of the MVPA models together may provide an overall picture of the activity underlining the process at hand.

\section{Conclusions}

Using a passive paradigm, we probed several different neurolinguistic properties. Separating the data into different frequency bands and looking at ITPC, we found that,

Table 3. Table of significant clusters in the syntax condition sorted by time from the divergence point

\begin{tabular}{|c|c|c|c|c|c|c|c|c|}
\hline \multicolumn{9}{|c|}{ Syntax } \\
\hline Band & Peak (\%) & $\begin{array}{l}\text { Peak } \\
\text { SD (\%) }\end{array}$ & $\begin{array}{c}\text { Peak } \\
\text { time (ms) }\end{array}$ & $\begin{array}{l}\text { Cluster } \\
\text { start (ms) }\end{array}$ & $\begin{array}{l}\text { Cluster } \\
\text { end (ms) }\end{array}$ & $\begin{array}{c}\text { Cluster } \\
\text { length (ms) }\end{array}$ & $\begin{array}{l}\text { Cluster } \\
\text { mean (\%) }\end{array}$ & $\begin{array}{l}\text { Cluster } \\
\text { SD (\%) }\end{array}$ \\
\hline$\gamma$-Low & 69.22 & 13.05 & 90 & 85 & 98 & 12 & 62.57 & 9.16 \\
\hline$\gamma$-Low & 68.36 & 11.57 & 302 & 300 & 314 & 14 & 64.04 & 5.10 \\
\hline
\end{tabular}

See the legend of Table 1 for an explanation of the columns.

Table 4. Table of peak scores for each bands and condition, dash (-) indicates no significant cluster

\begin{tabular}{|c|c|c|c|c|c|c|c|c|c|}
\hline \multirow[b]{2}{*}{ Band } & \multicolumn{3}{|c|}{ Lexical condition } & \multicolumn{3}{|c|}{$\begin{array}{c}\text { Condition } \\
\text { Semantic condition }\end{array}$} & \multicolumn{3}{|c|}{ Syntax condition } \\
\hline & $\begin{array}{l}\text { Peak } \\
\text { score }\end{array}$ & $\begin{array}{l}\text { Peak } \\
\text { SD }\end{array}$ & $\begin{array}{l}\text { Peak } \\
\text { time }\end{array}$ & $\begin{array}{l}\text { Peak } \\
\text { score }\end{array}$ & $\begin{array}{l}\text { Peak } \\
\text { SD }\end{array}$ & $\begin{array}{l}\text { Peak } \\
\text { time }\end{array}$ & $\begin{array}{l}\text { Peak } \\
\text { score }\end{array}$ & $\begin{array}{l}\text { Peak } \\
\text { SD }\end{array}$ & $\begin{array}{l}\text { Peak } \\
\text { time }\end{array}$ \\
\hline$\alpha$ & - & - & - & 91.11 & 12.96 & 106 & - & - & - \\
\hline$\beta$ & 85.97 & 14.95 & 538 & 75.00 & 19.08 & 138 & - & - & - \\
\hline$\gamma$-Low & 94.35 & 4.50 & 224 & 84.58 & 9.01 & 224 & 69.22 & 13.05 & 90 \\
\hline$\gamma$-Medium & 88.53 & 6.43 & 66 & - & - & - & - & - & - \\
\hline$\gamma$-High & 87.88 & 9.29 & 358 & - & - & - & - & - & - \\
\hline
\end{tabular}

Peak score is highest ROC-AUC scores of the cluster. Peak SD is the SD of cross-validation folds for the peak ROC-AUC score. Peak time is the time of the peak from DP. 
by using MVPA, we could classify lexical, semantic, and syntactic information processing. The best classification results varied between the different neurolinguistic properties both in time and, importantly, frequency bands, with lexical processes classified predominantly by broad $\gamma$, semantic distinctions by $\alpha$ and $\beta$, and morphosyntax by low $\gamma$ feature patterns. Crucially, all types of processing commenced in a near-parallel fashion from $\sim 100 \mathrm{~ms}$ after the auditory information allowed for disambiguating the spoken input. This shows that individual neurolinguistic processes take place near-simultaneously and involve overlapping yet distinct neuronal networks that operate at different frequency bands. Further investigations are needed to understand the precise relation of the time courses, frequency bands, neuronal substrates and neurolinguistic properties, and to test the applicability of this approach to detecting linguistic anomalies in various populations.

\section{References}

Baillet S, Mosher JC, Leahy RM (2001) Electromagnetic brain mapping. IEEE Signal Process Mag 18:14-30.

Bakker I, Takashima A, van Hell JG, Janzen G, McQueen JM (2015) Changes in theta and beta oscillations as signatures of novel word consolidation. J Cogn Neurosci 27:1286-1297.

Bastiaansen M, Hagoort P (2006). Oscillatory neuronal dynamics during language comprehension. In: Event-related dynamics of brain oscillations (Neuper C, Klimesch W, eds), pp 179-196. Amsterdam: Elsevier.

Bozic M, Fonteneau E, Su L, Marslen-Wilson WD (2015) Grammatical analysis as a distributed neurobiological function. Hum Brain Mapp 36:1190-1201.

Brennan J, Lignos C, Embick D, Roberts TPL (2014) Spectrotemporal correlates of lexical access during auditory lexical decision. Brain Lang 133:39-46.

Buzsáki G, Silva FL (2012) High frequency oscillations in the intact brain. Prog Neurobiol 98:241-249.

Buzsáki G, Schomburg EW (2015) What does gamma coherence tell us about inter-regional neural communication? Nat Neurosci 18: 484-489.

Bzdok D, Altman N, Krzywinski M (2018) Statistics versus machine learning. Nat Methods 15:233.

Canavier CC (2015) Phase-resetting as a tool of information transmission. Curr Opin Neurobiol 31:206-213.

Cannon J, McCarthy MM, Lee S, Lee J, Börgers C, Whittington MA, Kopell N (2014) Neurosystems: brain rhythms and cognitive processing. Eur J Neurosci 39:705-719.

Canolty RT, Edwards E, Dalal SS, Soltani M, Nagarajan SS, Kirsch HE, Berger MS, Barbaro NM, Knight RT (2006) High gamma power is phase-locked to theta oscillations in human neocortex. Science 313:1626-1628.

Cohen MX (2014) Analyzing neural time series data: theory and practice. Cambridge, MA: The MIT Press.

Dalal SS, Vidal JR, Hamamé CM, Ossandón T, Bertrand O, Lachaux JP, Jerbi K (2011) Spanning the rich spectrum of the human brain: slow waves to gamma and beyond. Brain Struct Funct 216:77-84.

Dale AM, Fischl B, Sereno MI (1999) Cortical surface-based analysis. I. Segmentation and surface reconstruction. Neuroimage 9:179-194.

Edmonds BA, Krumbholz K (2014) Are interaural time and level differences represented by independent or integrated codes in the human auditory cortex? J Assoc Res Otolaryngol 15:103-114.

Friederici AD (2002) Towards a neural basis of auditory sentence processing. Trends Cogn Sci 6:78-84.

Friederici AD (2011) The brain basis of language processing: from structure to function. Physiol Rev 91:1357-1392.
Friederici AD, Pfeifer E, Hahne A (1993) Event-related brain potentials during natural speech processing: effects of semantic, morphological and syntactic violations. Cognitive Brain Res 1:183-192.

Fries P (2005) A mechanism for cognitive dynamics: neuronal communication through neuronal coherence. Trends Cogn Sci 9:474-480.

Gansonre C, Højlund A, Leminen A, Bailey C, Shtyrov Y (2018) Task-free auditory EEG paradigm for probing multiple levels of speech processing in the brain. Psychophysiology 55:e13216.

Gramfort A, Luessi M, Larson E, Engemann DA, Strohmeier D, Brodbeck C, Goj R, Jas M, Brooks T, Parkkonen L, Hämäläinen M (2013) MEG and EEG data analysis with MNE-Python. Frontiers in neuroscience 7:267.

Gunter TC, Friederici AD, Schriefers H (2000) Syntactic gender and semantic expectancy: ERPs reveal early autonomy and late interaction. J Cogn Neurosci 12:556-568.

Haarmann H, Cameron K (2005) Active maintenance of sentence meaning in working memory: evidence from EEG coherences. Int J Psychophysiol 57:115-128.

Hagoort P (2004) Integration of word meaning and world knowledge in language comprehension. Science 304:438-441.

Hagoort P (2008) The fractionation of spoken language understanding by measuring electrical and magnetic brain signals. Philos Trans R Soc Lond B Biol Sci 363:1055-1069.

Hagoort P, Brown C (2000) ERP effects of listening to speech: semantic ERP effects. Neuropsychologia 38:1518-1530.

Hari R, Joutsiniemi SL, Sarvas J (1988) Spatial resolution of neuromagnetic records: theoretical calculations in a spherical model. Electroencephalogr Clin Neurophysiol 71:64-72.

Hasting AS, Kotz SA, Friederici AD (2007) Setting the stage for automatic syntax processing: the mismatch negativity as an indicator of syntactic priming. J Cogn Neurosci 19:386-400.

Haufe S, Meinecke F, Görgen K, Dähne S, Haynes J-D, Blankertz B, Bießmann $F$ (2014) On the interpretation of weight vectors of linear models in multivariate neuroimaging. Neuroimage 87:96-110.

Hauk O, Shtyrov Y, Pulvermüller F (2008) The time course of action and action-word comprehension in the human brain as revealed by neurophysiology. J Physiol Paris 102:50-58.

Herrmann B, Maess B, Friederici AD (2011) Violation of syntax and prosody-disentangling their contributions to the early left anterior negativity (ELAN). Neurosci Lett 490:116-120.

Hickok G, Poeppel D (2007) The cortical organization of speech processing. Nat Rev Neurosci 8:393-402.

Jas M, Engemann DA, Bekhti Y, Raimondo F, Gramfort A (2017) Autoreject: automated artifact rejection for MEG and EEG data. Neuroimage 159:417-429.

Kösem A, van Wassenhove V (2017) Distinct contributions of lowand high-frequency neural oscillations to speech comprehension. Lang Cogn Neurosci 32:536-544.

Kutas M, Hillyard SA (1980) Reading senseless sentences: brain potentials reflect semantic incongruity. Science 207:203-205.

Laureys S, Owen AM, Schiff ND (2004) Brain function in coma, vegetative state, and related disorders. Lancet Neurol 3:537-546.

Leiberg S, Lutzenberger W, Kaiser J (2006) Effects of memory load on cortical oscillatory activity during auditory pattern working memory. Brain Res 1120:131-140.

Levy J, Hagoort P, Démonet J-F (2014) A neuronal gamma oscillatory signature during morphological unification in the left occipitotemporal junction. Hum Brain Mapp 35:5847-5860.

Lewis AG, Wang L, Bastiaansen M (2015) Fast oscillatory dynamics during language comprehension: unification versus maintenance and prediction? Brain Lang 148:51-63.

Lopes da Silva FH (2006) Event-related neural activities: what about phase? Prog Brain Res 159:3-17.

Luo H, Poeppel D (2007) Phase patterns of neuronal responses reliably discriminate speech in human auditory cortex. Neuron 54:1001-1010.

Luo H, Poeppel D (2012) Cortical oscillations in auditory perception and speech: evidence for two temporal windows in human auditory cortex. Front Psychol 3. 
Lutzenberger W, Pulvermüller F, Birbaumer N (1994) Words and pseudowords elicit distinct patterns of $30-\mathrm{Hz}$ EEG responses in humans. Neurosci Lett 176:115-118.

MacGregor LJ, Pulvermüller F, van Casteren M, Shtyrov Y (2012) Ultra-rapid access to words in the brain. Nat Commun 3:711.

Majerus S, Bruno M-A, Schnakers C, Giacino JT, Laureys S (2009) The problem of aphasia in the assessment of consciousness in brain-damaged patients. Prog Brain Res 177:49-61.

Mamashli F, Khan S, Obleser J, Friederici AD, Maess B (2019) Oscillatory dynamics of cortical functional connections in semantic prediction. Hum Brain Mapp 40:1856-1866.

Marslen-Wilson WD (1987) Functional parallelism in spoken wordrecognition. Cognition 25:71-102.

Moseley RL, Pulvermüller F, Shtyrov Y (2013) Sensorimotor semantics on the spot: brain activity dissociates between conceptual categories within 150 ms. Sci Rep 3:1928.

Münte TF, Heinze H-J, Matzke M, Wieringa BM, Johannes S (1998) Brain potentials and syntactic violations revisited: no evidence for specificity of the syntactic positive shift. Neuropsychologia 36: 217-226.

Näätänen R, Paavilainen P, Rinne T, Alho K (2007) The mismatch negativity (MMN) in basic research of central auditory processing: a review. Clin Neurophysiol 118:2544-2590.

Neville H, Nicol JL, Barss A, Forster KI, Garrett MF (1991) Syntactically based sentence processing classes: evidence from eventrelated brain potentials. J Cogn Neurosci 3:151-165.

Ojala M, Garriga GC (2010) Permutation tests for studying classifier performance. J Mach Learn Res 11:1833-1863.

Oldfield RC (1971) The assessment and analysis of handedness: The Edinburgh inventory. Neuropsychologia 9:97-113.

Osterhout L, Holcomb PJ, Swinney DA (1994) Brain potentials elicited by garden-path sentences: evidence of the application of verb information during parsing. J Exp Psychol Learn Mem Cogn 20: 786-803.

Palva JM, Palva S, Kalia K (2005) Phase synchrony among neuronal oscillations in the human cortex. J Neurosci 25:3962-3972.

Pedregosa F, Varoquaux G, Gramfort A, Michel V, Thirion B, Grisel O, Blondel M, Prettenhofer P, Weiss R, Dubourg V, Vanderplas J, Passos A, Cournapeau D, Brucher M, Perrot M, Duchesnay E (2011) Scikit-learn: machine learning in python. J Mach Learn Res 12:2825-2830.

Phipson B, Smyth GK (2010) Permutation P-values should never be zero: calculating exact $\mathrm{P}$-values when permutations are randomly drawn. Stat Appl Genet Mol Biol 9:Article39.

Pulvermüller F (2005) Brain mechanisms linking language and action. Nat Rev Neurosci 6:576-582.

Pulvermüller F, Shtyrov Y (2006) Language outside the focus of attention: the mismatch negativity as a tool for studying higher cognitive processes. Prog Neurobiol 79:49-71.

Pulvermüller F, Fadiga $L$ (2010) Active perception: sensorimotor circuits as a cortical basis for language. Nat Rev Neurosci 11:351-360.

Pulvermüller F, Preissl H, Lutzenberger W, Birbaumer N (1996) Brain rhythms of language: nouns versus verbs. Eur J Neurosci 8:937941.

Pulvermüller F, Kujala T, Shtyrov Y, Simola J, Tiitinen H, Alku P, Alho K, Martinkauppi S, Ilmoniemi RJ, Näätänen R (2001) Memory traces for words as revealed by the mismatch negativity. Neuroimage 14:607-616.

Pulvermüller F, Huss M, Kherif F, Moscoso del Prado Martin F, Hauk O, Shtyrov Y (2006) Motor cortex maps articulatory features of speech sounds. Proc Natl Acad Sci USA 103:7865-7870.

Puolivali T, Palva S, Palva JM (2018) Influence of multiple hypothesis testing on reproducibility in neuroimaging research. bioRxiv 488353.

Ragazzoni A, Grippo A, Tozzi F, Zaccara G (2000) Event-related potentials in patients with total locked-in state due to fulminant Guillain-Barré syndrome. Int J Psychophysiol 37:99-109.

Sauseng P, Klimesch W (2008) What does phase information of oscillatory brain activity tell us about cognitive processes? Neurosci Biobehav Rev 32:1001-1013.
Sekihara K, Nagarajan SS (2008) Adaptive spatial filters for electromagnetic brain imaging. Berlin: Springer.

Shtyrov Y (2010) Automaticity and attentional control in spoken language processing: neurophysiological evidence. Ment Lex 5:255-276.

Shtyrov Y, Lenzen M (2017) First-pass neocortical processing of spoken language takes only $30 \mathrm{msec}$ : electrophysiological evidence. Cogn Neurosci 8:24-38.

Shtyrov Y, Hauk O, Pulvermüller F (2004) Distributed neuronal networks for encoding category-specific semantic information: the mismatch negativity to action words. Eur J Neurosci 19:10831092.

Shtyrov Y, Pihko E, Pulvermüller F (2005) Determinants of dominance: is language laterality explained by physical or linguistic features of speech? Neuroimage 27:37-47.

Shtyrov Y, Kujala T, Pulvermüller F (2010) Interactions between language and attention systems: early automatic lexical processing? J Cogn Neurosci 22:1465-1478.

Shtyrov Y, Butorina A, Nikolaeva A, Stroganova T (2014) Automatic ultrarapid activation and inhibition of cortical motor systems in spoken word comprehension. Proc Natl Acad Sci USA 111: E1918-E1923.

Shtyrov Y, Smith ML, Horner AJ, Henson R, Nathan PJ, Bullmore ET, Pulvermüller $F$ (2012) Attention to language: novel MEG paradigm for registering involuntary language processing in the brain. Neuropsychologia 50:2605-2616.

Tavabi K, Embick D, Roberts TPL (2011) Spectral-temporal analysis of cortical oscillations during lexical processing. Neuroreport 22: $474-478$.

Teng X, Tian X, Rowland J, Poeppel D (2017) Concurrent temporal channels for auditory processing: oscillatory neural entrainment reveals segregation of function at different scales. PLoS Biol 15: e2000812.

Thielscher A, Antunes A, Saturnino GB (2015). Field modeling for transcranial magnetic stimulation: a useful tool to understand the physiological effects of TMS? Engineering in Medicine and Biology Society (EMBC), 2015 37th Annual International Conference of the IEEE, pp 222-225.

Towle VL, Yoon HA, Castelle M, Edgar JC, Biassou NM, Frim DM, Spire JP, Kohrman MH (2008) ECoG gamma activity during a language task: differentiating expressive and receptive speech areas. Brain 131:2013-2027.

Tyler LK, Marslen-Wilson W (2008) Fronto-temporal brain systems supporting spoken language comprehension. Philos Trans R Soc Lond B Biol Sci 363:1037-1054.

Van Veen BD, Van Drongelen W, Yuchtman M, Suzuki A (1997) Localization of brain electrical activity via linearly constrained minimum variance spatial filtering. IEEE Trans Biomed Eng 44:867-880.

Varela F, Lachaux JP, Rodriguez E, Martinerie J (2001) The brainweb: phase synchronization and large-scale integration. Nat Rev Neurosci 2:229-239.

Varoquaux G, Raamana PR, Engemann DA, Hoyos-Idrobo A, Schwartz Y, Thirion B (2017) Assessing and tuning brain decoders: cross-validation, caveats, and guidelines. Neuroimage 145:166-179.

Von Stein A, Sarnthein J (2000) Different frequencies for different scales of cortical integration: from local gamma to long range alpha/theta synchronization. Int J Psychophysiol 38:301-313.

Vukovic N, Shtyrov Y (2014) Cortical motor systems are involved in second-language comprehension: evidence from rapid mu-rhythm desynchronisation. Neuroimage 102:695-703.

Wang L, Zhu Z, Bastiaansen M (2012) Integration or predictability? A further specification of the functional role of gamma oscillations in language comprehension. Front Psychol 3.

Westner BU (2017) The Hilbert beamformer pipeline. Retrieved from https://brittas-summerofcode.blogspot.com/2017/08/the-hilbertbeamformer-pipeline 29.html.

Westner BU, Dalal SS (2017) Faster than the brain's speed of light: retinocortical interactions differ in high frequency activity when processing darks and lights. bioRxiv 153551. 